\title{
Statistical simulation on microcomputers
}

\author{
DRAKE R. BRADLEY, MICHAEL W. SENKO, and FOSTER A. STEWART \\ Bates College, Lewiston, Maine
}

\begin{abstract}
The statistical simulation program DATASIM is designed to conduct large-scale sampling experiments on microcomputers. Monte Carlo procedures are used to investigate the Type I and Type II error rates for statistical tests when one or more assumptions are systematically violatedassumptions, for example, regarding normality, homogeneity of variance or covariance, minimum expected cell frequencies, and the like. In the present paper, we report several initial tests of the data-generating algorithms employed by DATASIM. The results indicate that the uniform and standard normal deviate generators perform satisfactorily. Furthermore, KolmogorovSmirnov tests show that the sampling distributions of $z, t, F, \chi^{2}$, and $r$ generated by DATASIM simulations follow the appropriate theoretical distributions. Finally, estimates of Type I error rates obtained by DATASIM under various patterns of violations of assumptions are in close agreement with the results of previous analytical and empirical studies. These converging lines of evidence suggest that DATASIM may well prove to be a reliable and productive tool for conducting statistical simulation research.
\end{abstract}

Although statistical simulations have traditionally been conducted on mainframe or minicomputers, the increasing availability of powerful yet inexpensive personal computers provides another tool for researchers in this area. A laboratory equipped with 20 to 30 microcomputers has computing power roughly comparable to a minicomputer running in a timesharing environment. Although the individual microcomputer is unable to match the minicomputer in sheer number-crunching performance, the availability of multiple processors allows many simulations to be run concurrently. The microcomputer's disadvantage in processing speed is therefore largely offset by an advantage in distributed processing. An additional benefit is that microcomputers allow accurate real-time control of stimulus and response events for conducting on-line research, whereas timesharing systems are necessarily deficient in this respect. Furthermore, the linking of microcomputers to a network can provide the same access to centralized resources (software, databases, communications, traffic logging, etc.) that is available with timesharing systems.

We have 25 PCs networked together that students use to conduct on-line research and statistical simulations. ${ }^{1}$ A general purpose data simulator is used to perform the statistical simulations. The software, called DATASIM (Bradley, 1988), is designed to generate large numbers of randomized datasets from populations specified by the user, and to "capture" and analyze the sampling distributions of statistics computed on the individual datasets. ${ }^{2}$ This provides a convenient way to conduct sampling experiments investigating the effects of violating assumptions on the Type I or Type II error rates of statistical tests (Bradley, 1989a, 1989b).

Correspondence may be addressed to Drake R. Bradley, Department of Psychology, Bates College, Lewiston, ME 04240.
Using a networked version of DATASIM, we have replicated a number of Monte Carlo studies (Boneau, 1960; Bradley \& Cutcomb, 1977; Collier, Baker, Mandeville, \& Hayes, 1967; Hsu \& Feldt, 1969; Norton, 1952) and analytical studies (Box, 1954; Hsu, 1938; Scheffé, 1959) that investigated the effects of violating assumptions on the Type I error rate. Generally, each such replication requires one overnight session on 20 or so PCs in the lab. For example, all of the sampling experiments reported by Norton (1952) can be replicated in one night. This performance is better than that achieved in previous years (e.g., Bradley, Bradley, McGrath, \& Cutcomb, 1979) when simulations were run at night (in batch) on a mainframe timesharing system.

The purpose of the present paper is twofold. First, we hope to illustrate the feasibility of conducting large-scale statistical simulations on microcomputers-an activity normally associated with larger machines. We will do this by demonstrating a number of simulations that can be conducted with DATASIM on an ordinary IBM-compatible PC. Second, we will show that the random number algorithms employed by DATASIM generate data with the desired theoretical properties, and that the software is therefore appropriate for statistical simulation research. We do this by examining DATASIM's uniform and standard normal deviate generators, by comparing the sampling distributions of statistics generated through simulation $\left(z, t, F, \chi^{2}, r\right)$ to their corresponding theoretical distributions, and by examining the extent to which DATASIM estimates of Type I error rates replicate the results of previous analytical and empirical studies. ${ }^{3}$

\section{UNIFORM RANDOM NUMBER GENERATION}

The quality of a Monte Carlo simulation hinges, in large part, on the uniform random number generator used 
by the program. DATASIM employs a well-known, thoroughly tested, and efficient algorithm for generating uniform random numbers in the interval $0-1$. The generator is a prime-modulus multiplicative congruential generator referred to as LLRANDOMII by Lewis and Orav (1989, pp. 88, 93). The algorithm is attributed to Lewis, Goodman, and Miller (1969):

$$
U_{i+1}=\left(16,807 U_{i}\right) \bmod \left(2^{31}-1\right)
$$

The quantity $U_{i}$ is the current value of the seed, an integer number between 1 and $2^{31}-2=2147483646$. The quantity $U_{i+1}$ is the next value to be generated in the sequence of pseudorandom numbers; it is computed by multiplying the current value of the seed $\left(U_{i}\right)$ by the multiplier 16,807 and taking the remainder that is left over when this product is divided by the prime modulus, $2^{31}-1=$ 2147483647. As with $U_{i}$, the value of $U_{i+1}$ will be an integer number between 1 and $2^{31}-2$. Dividing $U_{i+1}$ by the prime modulus $\left(2^{31}-1\right)$ will therefore produce a uniform random number $U$, in the interval $0<U<1$. Pseudocode for implementing the generator is as follows:

$$
\begin{aligned}
& \text { SEED }=\bmod (16807 * \text { SEED, } 2147483647) \\
& \text { RAND }=\text { SEED } / 2147483647
\end{aligned}
$$

In most contexts, SEED would be either a global or a static program variable, and RAND would define the returned value of a function. The initial value of SEED is either selected at random, or explicitly set by the user. Subsequent calls to the RAND function update the value of SEED and return a uniform random number based on this value. This procedure results in a sequence of pseudorandom numbers which can be regenerated at any time by reseeding the generator with the original starting value. DATASIM provides a variety of ways to set the initial value of SEED, as illustrated below.

Thorough testing of DATASIM's uniform randonı number generator would require subjecting its uniform random numbers to a variety of tests for uniformity, independence, high-dimensional structure, and so forth (Lehman, 1977, pp. 132-140; Lewis \& Orav, 1989, pp. 94-95). Since the LLRANDOMII generator used by DATASIM has been documented and tested by others (Learmonth, 1976; Learmonth \& Lewis, 1974), we will illustrate only the more obvious and straightforward tests here. The LLRANDOMII generator has been used in many other software packages, such as SPSS, APL, and the IMSL Library (see Lewis \& Orav, 1989, p. 85).

As noted elsewhere (Bradley, 1989a, 1989b), DATASIM is a command-driven software package in which individual commands or chains of commands are entered to initialize simulation parameters, to conduct simulations, and to analyze the results. Data are generated from distributions defined by the user: discrete or continuous, normal or non-normal, independent or correlated. However, all such data are ultimately based on uniform random numbers generated by the LLRANDOMII generator. For testing purposes, DATASIM permits the user to generate uniform random numbers directly. For example, suppose we would like to generate two groups of 5,000 uniform random numbers. The following commands illustrate one way to do this:

\section{DESIGN TWOGROUP, NOBS 5000 \\ UNIFORM 972183899}

The DESIGN command specifies a twogroup design, NOBS sets the sample size to $n=5,000$ for each group, and UNIFORM generates uniform random numbers using an initial seed of $U_{1}=972183899$. The initial value of the seed was selected at random from the range of possible values $\left(1\right.$ to $\left.2^{31}-2\right)$. The two samples of uniform random numbers are stored in columns $\mathrm{C} 1$ and $\mathrm{C} 2$ of the database, respectively. We may now enter additional DATASIM commands to display and analyze the data. For example, entering HIST $\mathrm{C} 1$ and HIST $\mathrm{C} 2$ produces the histograms of the uniform random numbers shown in Figures 1 and 2, respectively. The random numbers in both columns appear to be uniformly distributed throughout the interval $0-1$. Since the class interval size for the histograms is $i=.10$ (with midpoints at $.05, .15, \ldots$,

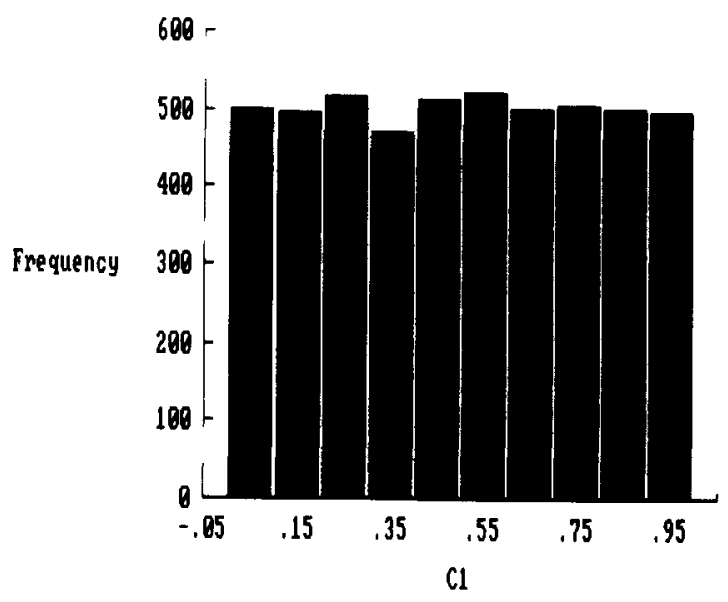

Figure 1. Histogram of the $n=5,000$ uniform random numbers in column 1.

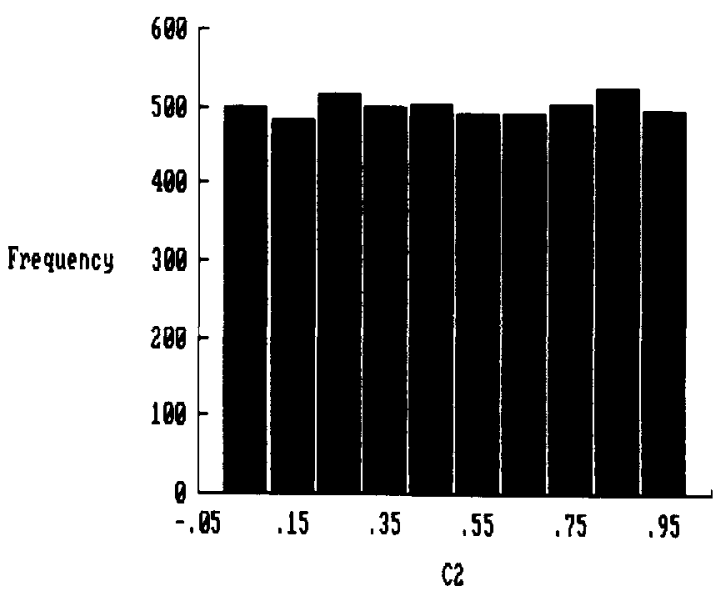

Figure 2. Histogram of the $n=5,000$ uniform random numbers in column 2. 
.95), the expected frequency for each interval is 500 . Goodness of fit $\left(\chi^{2}, 9 d f\right)$ tests verified that the distribution of observed frequencies did not differ significantly from expectation for either $\mathrm{Cl}$ or $\mathrm{C} 2(p>.20)$. To assess independence, the uniform random numbers in $\mathrm{C} 2$ were plotted against those in $\mathrm{Cl}$ by entering the command PLOT $\mathrm{C} 2 \mathrm{C1}$. The resulting scatterplot is shown in Figure 3. If the numbers in $\mathrm{Cl}$ and $\mathrm{C} 2$ are uniformly and independently distributed, then the scatterplot will produce a square evenly filled by data points. As is evident from Figure 3 , this is the case. Of course, plots like that in Figure 3 are most often based on pairs of random numbers that are separated by a lag of 1 or 2 , rather than 5,000 . The LLRANDOMII generator produces data that are uniformly distributed in the square for these lags as well (Lewis \& Orav, 1989, pp. 32-37).

A more rigorous test of uniformity may be conducted by comparing the cumulative distribution function (CDF) of a sample of uniform random numbers to the corresponding theoretical CDF. The Kolmogorov-Smirnov test may be used to determine whether the empirical CDF conforms to some particular theoretical CDF (uniform, normal, $t, F, \chi^{2}$, etc.). There are four steps involved in conducting the test: First, we need to generate a distribution of numbers (uniform, normal, etc.) to be evaluated by the test. Second, we must obtain the empirical CDF of these numbers. This is done by sorting the numbers and then computing the proportion of scores that are less than or equal to each score value in the sorted distribution. Third, we need to obtain the theoretical CDF for the numbers. This is done by referencing an appropriate theoretical distribution and determining the area under it that is to the left of the score value: in a standard normal distribution, for example, $p=.975$ falls to the left of the score $z=1.96$. For a distribution that is uniform in the interval $0-1$, the theoretical CDF is obtained quite easily, since the cumulative proportion is simply the value

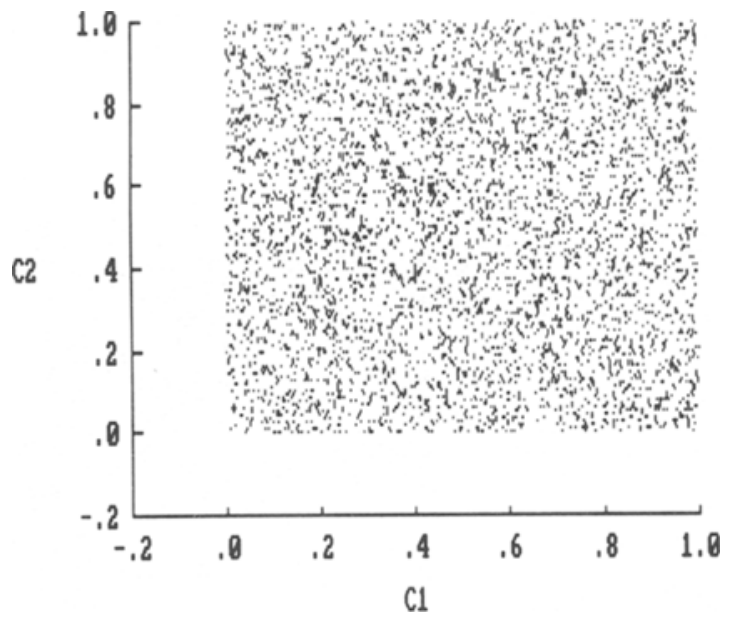

Figure 3. Scatterplot of the uniform random numbers in $\mathrm{C2}$ versus $\mathrm{C} 1$.

of the uniform random number itself: $p=.7534$, for example, would fall to the left of $U=.7534$. (Of course, the empirically determined cumulative proportion for $U=$ .7534 might be $p=.7359$ or some other value.) Finally, we conduct the Kolmogorov-Smirnov test. This is done by computing the statistic $D$, the maximum absolute difference between the empirical and theoretical cumulative proportions across all $n$ score values that were generated. If the score values do indeed follow the prescribed distribution, then the empirical and theoretical CDFs will be in close agreement, and the value of $D$ will be relatively small.

We now illustrate testing the CDF of a sample of DATASIM uniform random numbers. The following command list generates a single sample of $n=1,000$ uniform random numbers from an initial seed, selected at random, of $U_{1}=1497539941$ :

\section{DESIGN ONEGROUP, NOBS 1000 UNIFORM 1497539941}

Execution of these commands causes 1,000 uniform random numbers to be generated and stored in $\mathrm{Cl}$ of the data buffer. For uniform numbers, we must compute the empirical and theoretical CDFs prior to conducting the Kolmogorov-Smirnov test; for other distributions, $(t, F$, etc.) DATASIM will perform these calculations for us. The following command list computes the empirical CDF:

$$
\begin{aligned}
& \text { NCOL 3, SORT C1, SEQUENCE C2 } \\
& \text { LET C2 }=(\mathrm{C} 2 / 1000)-.0005
\end{aligned}
$$

The NCOL command redimensions the database to have three columns, so that we have room to store the CDFs in $\mathrm{C} 2$ and $\mathrm{C} 3$. The SORT command sorts the uniform random numbers in $\mathrm{Cl}$ into ascending order. The SEQUENCE command puts a sequence of numbers from 1 to 1,000 in $\mathrm{C} 2$, and the subsequent LET command converts these to cumulative proportions. (Note that .0005 is subtracted in order to place half of each score above, and half below, its own value as the midpoint of the interval.) Following execution of this command, C2 stores the actual proportion of values falling at or below the value of the corresponding uniform number in $\mathrm{Cl}$; hence, $\mathrm{C} 2$ contains the empirical CDF. To obtain the theoretical $\mathrm{CDF}$, we simply enter:

$$
\text { LET C3 = C1 }
$$

This command simply copies the uniform random values in $\mathrm{Cl}$ to $\mathrm{C} 3$, since, as noted above, these constitute the appropriate theoretical cumulative proportions. The first five values in $\mathrm{Cl}$ to $\mathrm{C} 3$ are as follows:

$\mathrm{Cl}$

.001190309

.001375744

.005530310

.009190446

.009961912
$\mathrm{C} 2$

.000500000

.001500000

.002500000

.003500000

.004500000
C3

.001190309

.001375744

.005530310

.009190446

.009961912 
We are now ready to conduct the Kolmogorov-Smirnov test. This is done by entering the command $\mathrm{KS}$, which causes DATASIM to compute $D$ from the values in $C 2$ and $\mathrm{C} 3$, and to plot the sample and theoretical cumulative proportions in $\mathrm{C} 2$ and $\mathrm{C} 3$ against the score values in $\mathrm{C} 1$. Figure 4 shows the output of the KS command, which is simply a plot of the two CDFs. We see that $D=$ .01675 and $p>.20$, so there is no evidence of a significant departure from uniformity in the data. In fact, the sample and theoretical CDFs are so close that it is impossible to distinguish them individually.

Figures 1-4 and other tests suggest that the DATASIM uniform random number generator is quite satisfactory. While "better" multipliers exist than the one used by LLRANDOMII $(16,807)$, they have the disadvantage that their products with $U_{i}$ may be too large to be computed correctly on some computers (Lewis \& Orav, 1989, pp. 89, 91-92). In any event, DATASIM allows the user to define a different multiplier and/or prime modulus for the generator. This is done using the RMULT and RMOD commands. Given a prime modulus of $2^{31}-1$, Fishman and Moore (1985) identified the following five "best" multipliers out of more than 267 million that were tested:
(1) $950,706,376$
(2) $742,938,285$
(3) $1,226,874,159$
(4) $62,089,911$
(5) $1,343,714,438$

If the RMULT command is used to select one of these (or other) multipliers, the user should verify that no loss in precision occurs when evaluating large products with $U_{i}$. This problem is least likely to arise with Multiplier 4 $(62,089,911)$.

This section has focused exclusively on the quality of uniform random number generation in DATASIM. However, it is often the case that random numbers following

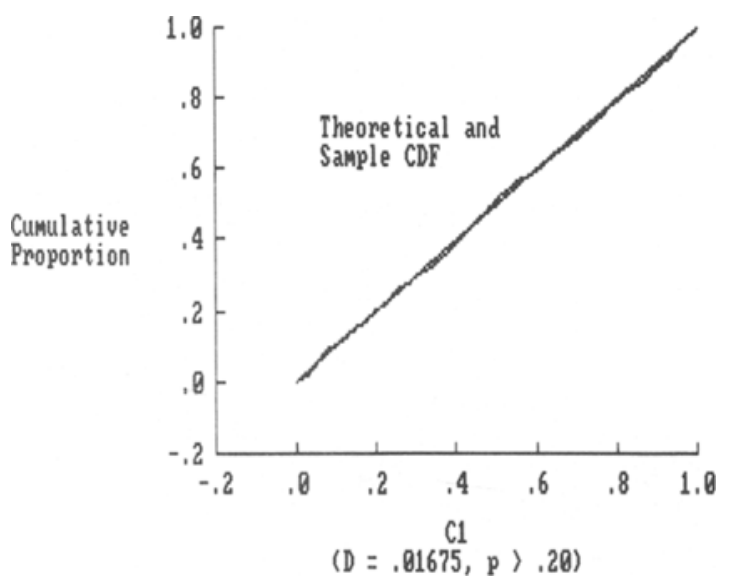

Figure 4. Sample and theoretical cumulative distribution functions for $n=1,000$ uniform random numbers. The sample uniforms were generated by seeding LLRANDOMII with an initial value of 1497539941. nonuniform distributions are desired. In general, such numbers can be obtained through appropriate transformations of uniform random numbers. One approach is to use uniform random numbers to define percentiles, and then use the inverse probability method (Lewis \& Orav, 1989 , p. 39) to obtain the corresponding values of $X$ in the distribution. Other approaches are often used because they are faster, more computationally feasible, or both. The Box-Muller algorithm (Box \& Muller, 1958), described below, is an example of one such alternative approach. In any case, a properly functioning uniform random number generator is a necessary, but not sufficient, condition for the resulting nonuniform random numbers to be distributed properly. In the next section, we examine how well DATASIM generates data for one very important nonuniform distribution, the standard normal.

\section{STANDARD NORMAL DEVIATE GENERATION}

Unless otherwise specified by the user, DATASIM will generate data following a normal distribution. Standard normal deviates are generated two at a time, using the Box-Muller algorithm (Box \& Muller, 1958; Lewis \& Orav, 1989, p. 45):

$$
\begin{aligned}
& z_{1}=\sqrt{\left(-2 \cdot \log _{e} U_{1}\right)} \cdot \cos \left(2 \pi U_{2}\right) \\
& z_{2}=\sqrt{\left(-2 \cdot \log _{e} U_{1}\right)} \cdot \sin \left(2 \pi U_{2}\right)
\end{aligned}
$$

With this algorithm, two standard normal deviates $\left(z_{1}\right.$ and $z_{2}$ ) are generated for each pair of uniform random numbers $\left(U_{1}\right.$ and $\left.U_{2}\right)$ fetched from the LLRANDOMII generator. Once generated, the $z$ scores are scaled and rounded to produce the $X$ scores desired by the user. Correlated $z$ scores may be generated as well, if needed (Bradley, 1989 b, p. 169).

To test the Box-Muller algorithm, the following DATASIM commands are entered to generate a single column of $n=1,000$ standard normal deviates:

\section{DESIGN ONEGROUP, NOBS 1000 \\ MU 0, SIGMA 1, DECI 10 \\ SEED 877846208}

Note that DESIGN and NOBS define the dimensions of the database, MU and SIGMA define the population parameters ( $\mu$ and $\sigma$ ) for a standard normal distribution, and DECI 10 tells DATASIM to generate scores with 10 decimal places of precision. The SEED command initializes the random number generator with $U_{1}=877846208$, and then generates and stores the 1,000 standard normal deviates in $\mathrm{Cl}$.

Figure 5 shows the output of the PDF $\mathrm{Cl}$ command, which displays the theoretical distribution (in this case a standard normal) employed to generate the simulated data. The PDF may be compared to a histogram of the simulated data (HIST C1) in order to assess the match between the theoretical and empirical distributions. However, a more rigorous comparison can be provided through examination of the empirical and theoretical CDFs. DATASIM can make this comparison directly, provided that the 


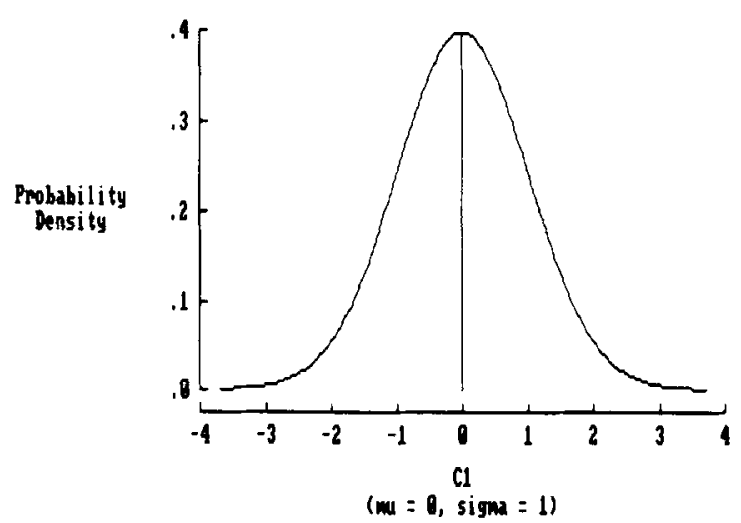

Figure 5. Theoretical distribution for generating standard normal deviates.

$z$ scores in $\mathrm{Cl}$ are stored in an external file. They may then be processed directly by an extended version of the KS command designed to process data stored on disk. The following commands perform the required operations:

\section{COPY DATA ZDIST}

\section{KS ZDIST $\mathrm{C} 1 \mathrm{Z}$}

The COPY command copies the $z$ scores to the file ZDIST. The KS command then redimensions the database to have three columns, thereby making room for the empirical and theoretical CDFs in $\mathrm{C} 2$ and $\mathrm{C} 3$, respectively. Next, the KS command reads the $z$ scores in ZDIST into the first column of the DATASIM data buffer. (While this step may seem redundant, this procedure is necessary for processing sampling distributions, as shown below.) The KS command then sorts the $z$ scores in $\mathrm{Cl}$ of the data buffer, computes the sample cumulative proportions as illustrated above, and stores them in C2. Finally, the KS command computes the theoretical cumulative proportions for the $z$ scores in $\mathrm{Cl}$ and stores them in $\mathrm{C} 3$.

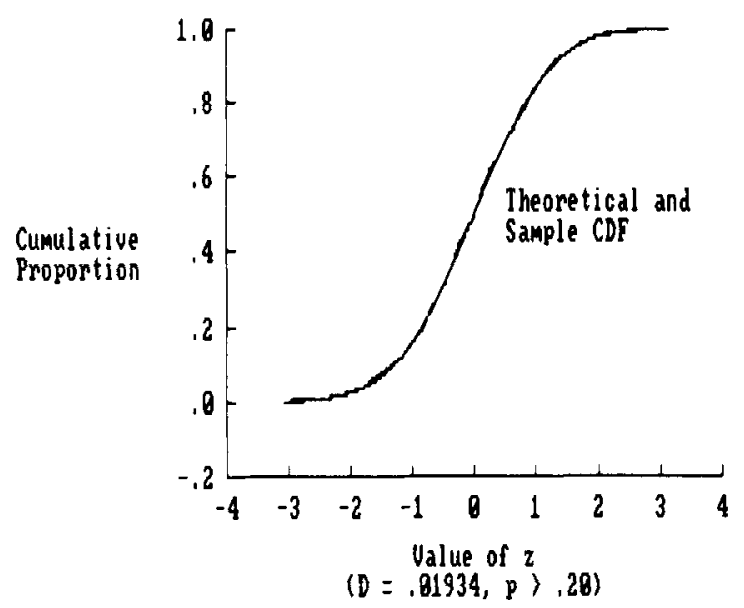

Figure 6. Sample and theoretical cumulative distribution functions for $n=1,000$ standard normal deviates. The deviates were obtained by applying the Box-Muller algorithm to LLRANDOMII uniforms initially seeded with 877846208 .
The token " $\mathrm{Z}$ " in the KS command tells DATASIM to compute the cumulative proportions with respect to a standard normal distribution. (For example, if the values $z=$ $-1.96, z=0.00$, and $z=1.96$ occur at various locations in $\mathrm{Cl}$, then the values $p=.025, p=.500$, and $p=$ .975 will be stored in the corresponding locations of $C 3$.) The empirical and theoretical CDFs are then plotted for comparison, as is shown in Figure 6. Once again, the sample and theoretical CDFs are indistinguishable, and the Kolmogorov-Smirnov test is insignificant: $D=.01934$, $p>.20$. This verifies that the Box-Muller algorithm is indeed generating normally distributed deviates, at least within the limits of the sensitivity of the KolmogorovSmirnov test. ${ }^{4}$

The results presented thus far show that DATASIM successfully generates uniform random numbers and standard normal deviates, in the sense that the resulting distributions conform to expectation. From various theorems in mathematical statistics, we can deduce that if the data generated by DATASIM follow a normal distribution, then the sampling distributions of various statistics computed on the data should follow known theoretical distributions (the $z, t, F$, or $\chi^{2}$ distributions, for example). This matter is examined in the next section.

\section{SAMPLING DISTRIBUTIONS OF STATISTICS}

As noted elsewhere (Bradley, 1988, 1989a, 1989b), DATASIM can generate and analyze sampling distributions for a variety of statistics. The extent to which these empirically generated sampling distributions match their theoretical counterparts is of particular concern to Monte Carlo researchers. This section evaluates the degree of fit between the empirical and theoretical distributions of $z, t, F, \chi^{2}$, and $r$.

\section{The $z$ Statistic}

We begin by assessing whether or not one-sample $z$ tests conducted on DATASIM-simulated datasets do in fact follow the standard normal distribution. The Appendix illustrates how to initialize the simulation, generate and analyze the simulated data, and determine whether the resulting sampling distribution has the proper form. The DESIGN and NOBS commands specify a one-group design with $n=3$ observations; MU and SIGMA define a standard normal distribution; and DECI tells DATASIM to retain 10 or more decimal places of precision in generating the data. Finally, the NLMU and NLSIG commands specify the values of $\mu$ and $\sigma$ under the null hypothesis $\left(H_{0}\right)$. These values are needed for calculating one-sample $z$ tests on the simulated datasets. Note that all of the commands entered to this point are required to initialize the simulation.

Following the initialization, we are in a position to perform repetitive simulation-that is, to generate and analyze datasets over and over again. When conducting large scale simulations, it is desirable to "capture" the simulation output so that the resulting sampling distributions 
can be processed by DATASIM. This is done by opening a file on disk for storing the results of the simulation. As is shown in the Appendix, the command OPEN ZDIST tells DATASIM to open the file ZDIST for output, and to write all subsequent program $\mathrm{I} / \mathrm{O}$ to this file as well as to the screen. (The screen can be closed for output, if desired, in order to speed up the simulation.) Following the OPEN command, the command list SIMULATE 10002067513105 , ONEZ instructs DATASIM to generate 1,000 simulated datasets, and to conduct a one-sample $z$ test on each. The value 2067513105 is the master seed used to initialize the uniform random number generator. ${ }^{5}$ The output generated by the SIMULATE command accumulates in the output file ZDIST until all 1,000 datasets have been generated and analyzed. To conserve space, the Appendix shows the output for just the first 8 datasets.

Following execution of the SIMULATE command, the output file is closed by the command CLOSE ZDIST. Program I/O is now directed only to the screen, and we may examine the sampling distribution of the $z$ statistics stored in ZDIST. However, because ZDIST contains a literal copy of the text output by the SIMULATE command, it is not in a form that can be directly analyzed by DATASIM. What is needed is a file that contains only numbers, or in the present case, the seeds, $z$ test values, and $p$ levels that were output for the simulated datasets. The command STRIP ZDIST performs the necessary editing by stripping ZDIST of all text and leaving only numeric values. In addition, STRIP consolidates all of the numeric values associated with a particular dataset (as coded by its initial seed) in one row of the file. As is shown in the Appendix, entering the command TYPE ZDIST displays the contents of the edited file: Each row consists of the seed, $z$ test value, and $p$ level for a particular simulated dataset. (Pressing the ESC key terminates the listing of the file.) Organized in this way, the numbers in the file may be processed directly with the KS command. The tokens

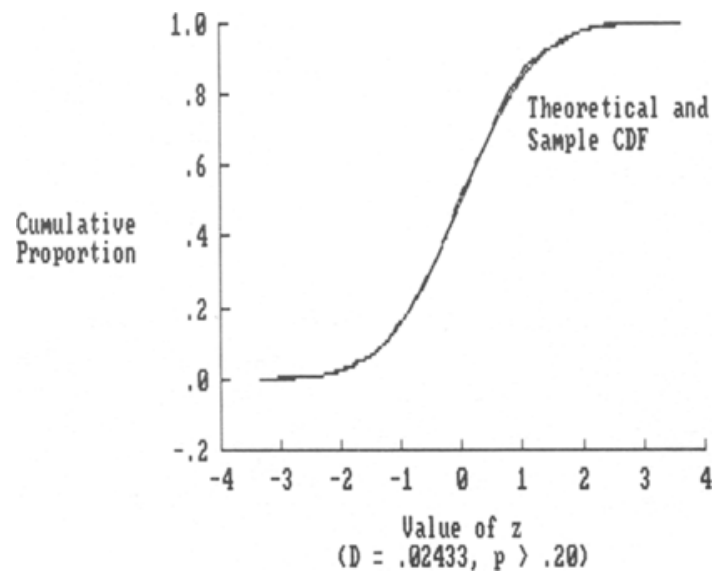

Figure 7. Sample and theoretical cumulative distribution functions for $N=1,000 z$ statistics. Each $z$ is computed on a random sample of $n=3$ standard normal deviates, and performs a one-sample test of $H_{0}: \mu=0$.
ZDIST C2 Z of the KS command (Appendix) tell DATASIM to compare the $z$ statistics stored in $C 2$ of the file ZDIST to a standard normal distribution $(Z)$. The output of the KS command is shown in Figure 7, where it may be seen that the maximum absolute difference between the CDFs is $D=.02433, p>.20$. As above, the sample and theoretical CDFs are too close to distinguish individually. This indicates that the sampling distribution of the $z$ statistics does in fact follow a standard normal distribution.

\section{The $t$ Statistic}

A similar approach is used to assess the sampling distribution of the $t$ statistic. For example, assume we want to conduct two-sample $t$ tests on a large number of simulated datasets. The commands for doing this are similar to those shown in the Appendix:

DESIGN TWOGROUP, NOBS 3
MU 0, SIGMA 1 , DECI 10
OPEN TDIST
SIMULATE 10001223512941 , TWOT
CLOSE TDIST
STRIP TDIST
KS TDIST C 2 T 4

In this case, DESIGN and NOBS define a two-group design with $n=3$ observations per group, and $M U$, SIGMA, and DECI specify standard normal deviates. The SIMULATE command generates 1,000 datasets, and TWOT conducts a two-sample $t$ test on each. The resulting output is stored in the file TDIST, the text stripped from the file, and a Kolmogorov-Smirnov test conducted. The tokens TDIST C2 T 4 in the KS command tell DATASIM to compare the $t$ statistics in C2 of TDIST to a $t$ distribution having $4 d f$. Following execution of the KS command, $\mathrm{Cl}$ of the DATASIM data buffer contains the sample $t$ statistics (in sorted order), C2 contains the sample cumulative proportions, and $\mathrm{C} 3$ contains the theoretical cumulative proportions. Note that each value in $\mathrm{C} 3$ is simply the area in a $t$ distribution $(d f=4)$ falling below the associated $t$ value in $\mathrm{Cl}$. The user may inspect the contents of these three columns by entering the command DISPLAY (press ESC to terminate the listing). The results of the KS analysis are presented in Figure 8. Again, there is a close correspondence between the sample and theoretical CDFs, with $D=.01780, p>.20$.

\section{The $F$ Statistic}

A minor variant on the preceding commands allows us to generate and test the sampling distribution of the $F$ statistic:

\author{
DESIGN ONEWAY 3 , NOBS 3 \\ MU 0, SIGMA 1, DECI 10 \\ OPEN FDIST \\ SIMULATE 1000 478534367, AOV 01 \\ CLOSE FDIST \\ STRIP FDIST
}

KS FDIST C2 F 26 
The DESIGN command specifies a one-way design with $k=3$ groups, and the AOV command conducts a oneway ANOVA on each simulated dataset (the 01 option forces brief output for the ANOVA). The tokens FDIST C2 F 26 in the KS command tell DATASIM to compare the $F$ statistics in C2 of the file FDIST to an $F$ distribution with 2 and $6 d f$. As above, the sorted $F$ values are put in $\mathrm{C} 1$, the sample cumulative proportions in $\mathrm{C} 2$, and the theoretical cumulative proportions in $\mathrm{C} 3$. The results are presented in Figure 9, where it can be seen that the theoretical and sample CDFs coincide with $D=.02411$, $p>.20$.

\section{The $\chi^{2}$ Statistic}

The tests reported so far verify that DATASIM generates sampling distributions of $z, t$, and $F$ which correspond closely to the theoretical distributions. We now report

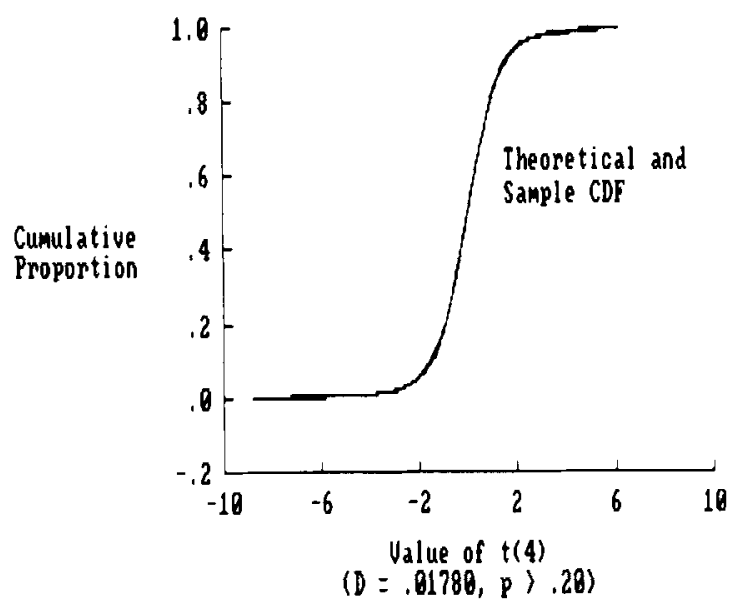

Figure 8. Sample and theoretical cumulative distribution functions for $N=1,000 t$ statistics ( $4 d f$ ). Each $t$ is computed on two random samples of $n=3$ standard normal deviates, and tests $H_{0}: \mu_{1}=\mu_{2}$.

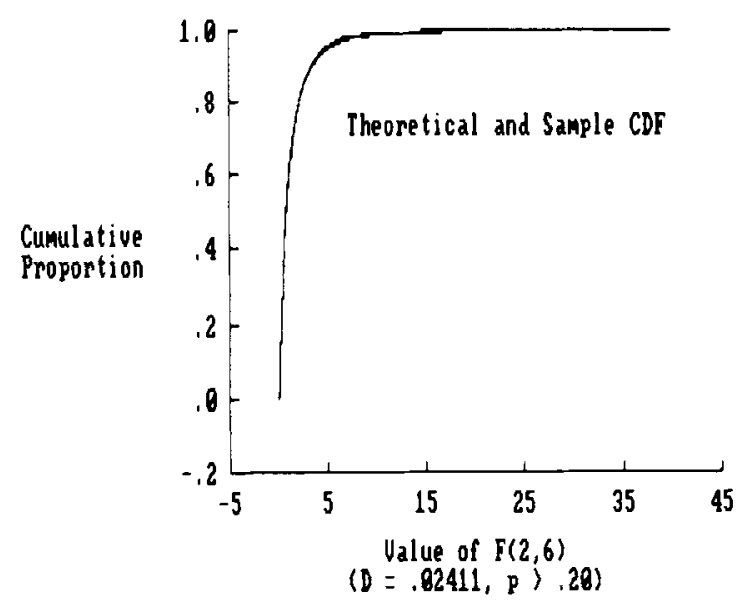

Figure 9. Sample and theoretical cumulative distribution functions for $N=1,000 F$ statistics (2/6 df). Each $F$ is computed on $k=3$ random samples of $n=3$ standard normal deviates, and tests $H_{0}$ : $\mu_{1}=\mu_{2}=\mu_{3}$. several tests comparing the $\chi^{2}$ statistic to the theoretical $\chi^{2}$ distribution. Note that in some applications of the $\chi^{2}$ test, the use of the $\chi^{2}$ distribution provides only an approximate solution, whereas in others it provides an exact solution. For instance, tests of independence or goodness of fit, as applied to discrete data, produce $\chi^{2}$ statistics that are only approximately distributed as $\chi^{2}$. On the other hand, the $\chi^{2}$ test used to compare a single sample variance $\left(s^{2}\right)$ to a known theoretical value $\left(\sigma^{2}\right)$ is distributed as $\chi^{2}$ exactly, provided that the assumptions of the test are met. Consequently, only in the latter case can we expect DATASIM to generate $\chi^{2}$ statistics that precisely follow the theoretical $\chi^{2}$ distribution. In the former case, the correspondence between the sample and theoretical distributions will depend on the sample size of the individual tables on which $\chi^{2}$ is computed.

Consider first the application of the $\chi^{2}$ test of independence to a $2 \times 2$ contingency table. The following commands provide for an assessment of the match between the sample and theoretical distributions:

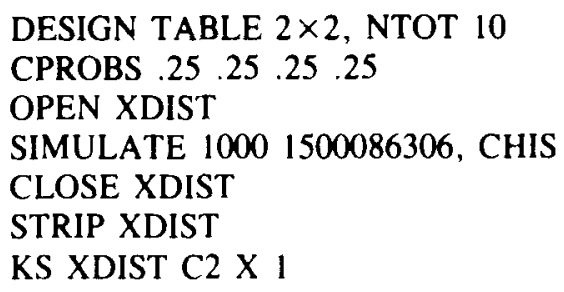

The DESIGN command specifies a $2 \times 2$ table design, NTOT sets the sample size for the table to $n=10$, and CPROBS assigns the probability of sampling an observation in each of the four cells of the table. The SIMULATE command generates 1,000 simulated datasets (2 $\times 2$ tables), and the CHIS command conducts a $\chi^{2}$ test of independence on each. The tokens XDIST C2 X 1 in the KS command tell DATASIM to compare the $\chi^{2}$ statistics in $\mathrm{C} 2$ of the file XDIST to a theoretical $\chi^{2}$ distribution with $1 d f$. The results are shown in Figure 10. In contrast to the previous examples, there is a detectable difference between the sample and theoretical CDFs, as verified by the Kolmogorov-Smirnov test: $D=.0695$, $p<.01$. This shows that the $\chi^{2}$ statistic computed for tests of independence on contingency tables is not, in fact, precisely distributed as $\chi^{2}$. Indeed, this is the rationale behind the various rules of thumb regarding minimum expected frequencies and the use of the $\chi^{2}$ test. Since the $\chi^{2}$ statistic for $2 \times 2$ tables is known to follow the multinomial distribution, the results shown in Figure 10 indicate that the Kolmogorov-Smirnov test is sensitive to real differences between the actual and hypothesized distributions.

Of course, the quality of the $\chi^{2}$ approximation improves as the sample size of the table increases. Figure 11 shows the results of the Kolmogorov-Smirnov analysis of a repetitive simulation employing $2 \times 2$ tables with $n=$ 40 observations, based on a master seed of 2100891807 . Although there is still some discrepancy between the sample and theoretical CDFs, it is not as large: $D=.04249$, 


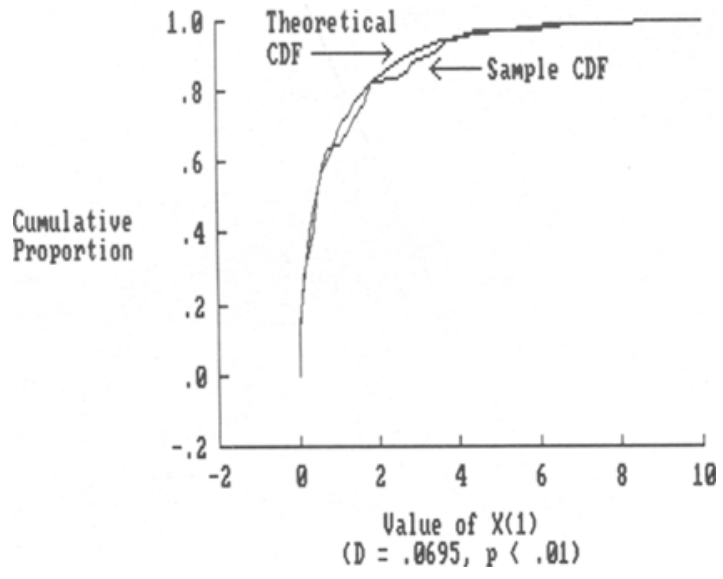

Figure 10. Sample and theoretical cumulative distribution functions for $N=1,000 \chi^{2}$ statistics (1 df). Each $\chi^{2}$ is computed on a $2 \times 2$ contingency table containing $n=10$ randomly sampled observations.

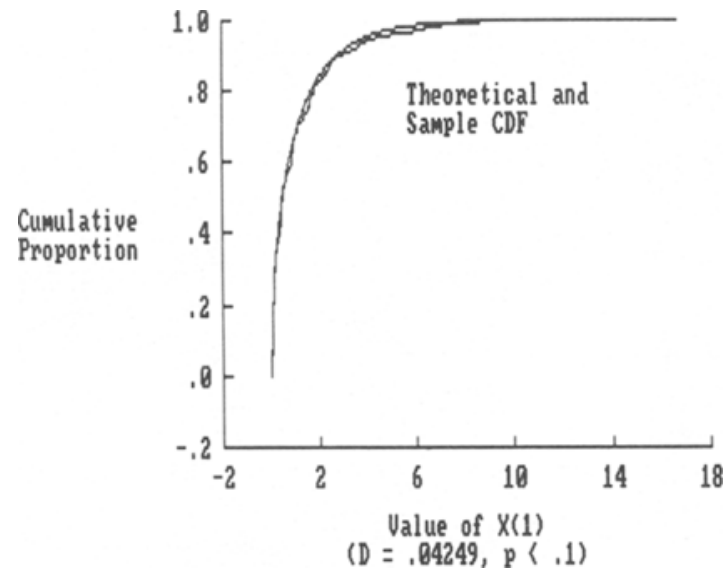

Figure 11. Sample and theoretical cumulative distribution functions for $N=1,000 \chi^{2}$ statistics ( $\left.1 d f\right)$. Each $\chi^{2}$ is computed on a $2 \times 2$ contingency table containing $n=40$ randomly sampled observations.

$p<.10$. The quality of the $\chi^{2}$ approximation improves rapidly with $n>40$. Nevertheless, the lack of correspondence between the sample and theoretical CDFs for small $n$ is not necessarily an argument against using the $\chi^{2}$ test. This is because the discrepancies in the distributions may not occur at the $1-\alpha=.95$ region of the $\mathrm{CDF}$, or may not be large enough to concern the researcher even if they do. This issue is examined in greater detail by Bradley et al. (1979).

The sampling distribution of the $\chi^{2}$ statistic as applied to tests of a single sample variance should conform precisely to the theoretical distribution. The following commands provide a test of this case:

DESIGN ONEGROUP, NOBS 2

MU 0, SIGMA 1, DECI 10, NLSIG 1

OPEN XDIST

SIMULATE 1000 969570515, XVAR

\section{CLOSE XDIST \\ STRIP XDIST \\ KS XDIST C2 X 1}

The DESIGN command specifies a onegroup design. To facilitate comparison with Figures 10 and 11, NOBS is set to $n=2$ in order to provide a $\chi^{2}$ test having $1 d f$. The MU, SIGMA, and DECI commands initialize a simulation for generating standard normal deviates, and NLSIG specifies the value of $\sigma$ under $H_{0}$. The SIMULATE command generates 1,000 simulated datasets, and XVAR conducts a $\chi^{2}$ test on each in order to test $H_{0}: \sigma^{2}=1$. The KS command compares the $\chi^{2}$ statistics stored in the file XDIST to a theoretical $\chi^{2}$ distribution with $1 d f$. The results are presented in Figure 12. The agreement between the sample and theoretical distributions is quite good, with a maximum absolute difference between the CDFs of only $D=.02790, p>.20$.

\section{The $r$ Statistic}

Finally, we assess the sampling distribution of the correlation coefficient, $r$, generated through repetitive simulation. The following commands perform the simulation and $\mathrm{KS}$ analysis:

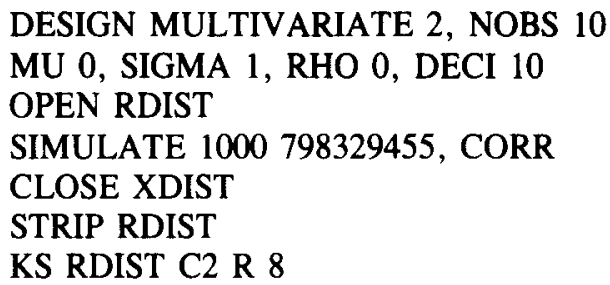

The DESIGN and NOBS commands specify a multivariate design with two variables having $n=10$ observations each, and MU, SIGMA, and DECI initialize a simulation for generating standard normal deviates. The RHO command specifies the degree of correlation in the population between the two variables ( 0 in this case). The

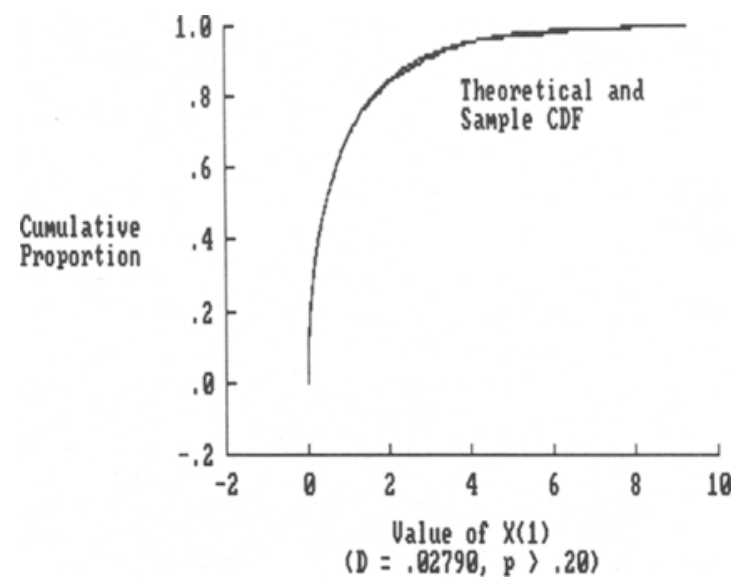

Figure 12. Sample and theoretical cumulative distribution functions for $N=1,000 \chi^{2}$ statistics ( $1 \mathrm{df}$ ). Each $\chi^{2}$ is computed on a single sample of $n=2$ standard normal deviates, and tests $H_{0}$ : $\sigma^{2}=1$. 


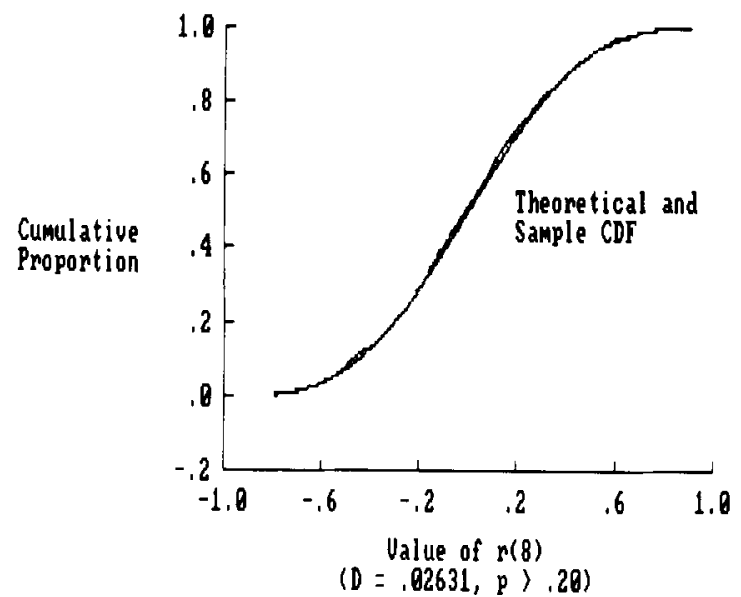

Figure 13. Sample and theoretical cumulative distribution functions for $N=1,000$ values of $r(8 d f)$. Each $r$ is computed on two random samples of $n=10$ standard normal deviates.

SIMULATE command generates 1,000 bivariate datasets, computes the correlation coefficient on each, and stores the results in the output file RDIST. The tokens RDIST C2 R 8 in the KS command tell DATASIM to compare the sampling distribution of $r$ in C2 of the file RDIST to a theoretical distribution of $r$ with $8 d f$. The results are presented in Figure 13. There is again a very close correspondence between the sample and theoretical CDFs, with a maximum discrepancy of only $D=.02631$, $p>.20$.

\section{Noncentral Distributions}

Each of the foregoing analyses compared the empirical sampling distribution of a test statistic to the corresponding theoretical distribution expected under $H_{0}-$ that is, to central $z, t, F, \chi^{2}$, or $r$. This is because each simulation was initialized so that $H_{0}$ was true. Similar comparisons may be made when simulations are initialized such that $H_{0}$ is false: for example, $\mu_{1} \neq \mu_{2}, \varrho \neq 0$, and so on. In this case, the empirical sampling distribution is compared to the appropriate noncentral distribution of the test statistic, as defined by the noncentrality parameter. At present, the KS command only calculates cumulative probabilities for central $z, t, F, \chi^{2}$, and $r$. However, the user can have DATASIM count the number of test statistics in the empirical distribution that fall within any of several intervals, convert these values to cumulative proportions, and then determine the corresponding theoretical values by referencing tables of the noncentral distribution. The Kolmogorov-Smirnov test can then be computed manually.

\section{REPLICATION OF PREVIOUS RESEARCH}

By explicitly comparing a variety of empirical and theoretical distributions, the preceding analyses have demonstrated that DATASIM generates datasets and sampling distributions that have the expected distribution properties. The Kolmogorov-Smirnov analyses are necessary to verify the accuracy of DATASIM as a general purpose statistical simulation program. If a data simulator is unable to pass these tests, its use for conducting Monte Carlo simulations is certainly called into question. In any event, the next step is to use DATASIM to replicate a number of previous analytical and empirical investigations. If DATASIM is able to reproduce the results obtained in previous studies, this would provide additional evidence regarding its reliability for statistical simulation research.

We are currently using DATASIM to replicate a number of well-known studies in the literature. Since the results of these replications will be presented in detail elsewhere, we summarize only the basic findings here. First, we have used DATASIM to conduct simulations assessing the Type I error rate for the $F$ test as applied to two or more samples drawn from populations with heterogeneous variances. Both equal and unequal sample sizes were employed. On the basis of the analytical work of Hsu (1938), Box (1954), and Scheffé (1959), we know the exact error rates for a variety of combinations of sample sizes and variance ratios (see Glass, Peckham, \& Sanders, 1972, for a review). In nearly all cases, the Type I error rates obtained by DATASIM simulations fall within the $95 \%$ confidence limits constructed about the exact values. However, there are a few cases in which the DATASIM estimates consistently differ from the exact values reported by Box (1954) and Hsu (1938). Faced with the possibility that the data simulator might be flawed, the exact values were recalculated. ${ }^{6}$ This revealed several errors in the values published in previous tables of exact Type I error rates. ${ }^{7}$ Since many of the calculations were originally performed without the assistance of a computer, these errors are understandable given the complexity of the analytical techniques employed to compute the "exact" values. In any event, the DATASIM Type I error estimates fall within the $95 \%$ confidence limits constructed about the recalculated exact values. These tests show, therefore, that DATASIM generates empirical Type I error rates that are in close agreement with the values predicted using analytical methods.

Another basis for testing DATASIM is provided by the results of previous empirical research. A number of Monte Carlo studies have been carried out to investigate the effects of non-normality, heterogeneity of variance, heterogeneity of covariance, limited scale values (e.g., dichotomous variables), and the like on the Type I error rate for $t$ and $F$. In some cases, several assumptions are violated simultaneously. These empirical investigations embody what is presently known about the consequences of violating assumptions on inflating or deflating the Type I error rate. We have now replicated a number of these studies, including the work of Boneau (1960), Collier et al. (1967), Hsu and Feldt (1969), and Norton (1952). So far, DATASIM has generated estimates of Type I error rates that are consistent with those reported in previous studies. ${ }^{8}$ 


\section{SUMMARY}

The utility of DATASIM as a general purpose statistical simulation program has been assessed in a variety of ways. First, the uniform and standard normal deviate generators were tested and found to have satisfactory performance. Second, Kolmogorov-Smirnov analyses were conducted on the sampling distributions of $z, t, F, \chi^{2}$, and $r$ obtained from simulation, and it was found that the empirical and theoretical distributions matched quite closely. The only exception consisted of $\chi^{2}$ tests of independence applied to $2 \times 2$ tables with small $n$. This is to be expected, since the $\chi^{2}$ test is only approximately distributed as $\chi^{2}$ in this application. Third, DATASIM successfully replicated the results of several previous analytical and empirical studies investigating the Type I error rate of $t$ and $F$ when assumptions are violated. Although additional tests remain to be done, these converging lines of evidence suggest that DATASIM holds considerable promise as a Monte Carlo research tool.

\section{REFERENCES}

Boneau, C. A. (1960). The effects of violations of assumptions underlying the $t$ test. Psychological Bulletin, 57, 49-64.

Box, G. E. P. (1954). Some theorems on quadratic forms applied in the study of analysis of variance problems: I. Effect of inequality of variance in the one-way classification. Annals of Mathematical Statistics, 25, 290-302.

Box, G. E. P., \& Muller, M. E. (1958). A note on the generation of random normal deviates. Annals of Mathematical Statistics, 29, 610-611.

Bradley, D. R. (1988). DATASIM. Lewiston, ME: Desktop Press. Bradley, D. R. (1989a). Computer simulation with DATASIM. Behavior Research Methods, Instruments, \& Computers, 21, 99-112.

Bradley, D. R. (1989b). A general purpose simulation program for statistics and research methods. In G. Garson \& S. Nagel (Eds.), $A d$ vances in social science and computers (Vol. 1, pp. 145-186). Greenwich, CT: JAI Press

Bradley, D. R., Bradley, T. D., McGrath, S. G., \& Cutcomb, S. D. (1979). Type I error rate of the chi-square test of independence in $\mathrm{R} \times \mathrm{C}$ tables that have small expected frequencies. Psychological Bulletin, 86, 1290-1297.

Bradley, D. R., \& CutcomB, S. (1977). Monte Carlo simulations and the chi-square test of independence. Behavior Research Methods and Instrumentation, 9, 193-201.

Collier, R. O., Baker, F. B., Mandeville, G. K., \& Hayes, T. F. (1967). Estimates of test size for several test procedures based on conventional variance ratios in the repeated measures design. Psychometrika, 32, 339-353.

Fishman, G., \& MOORE, L. (1985). An exhaustive analysis of multiplicative congruential random number generators with modulus $2^{31}-1$. SIAM Journal of Scientific \& Statistical Computing, 7, 24-45.

Glass, G. V., Peckham, P. D., \& SAnders, J. R. (1972). Consequences of failure to meet assumptions underlying the fixed effects analyses of variance and covariance. Review of Educational Research, 42, 237-288.

Hsu, P. L. (1938). Contribution to the theory of "Student's" $t$-test as applied to the problem of two samples. Statistical Research Memoirs, 2, 1-24.

Hsu, T. C., \& Feldt, L. S. (1969). The effect of limitations on the number of criterion score values on the significance level of the F-test. American Educational Research Joumal, 6, 515-527.

LEARMONTH, G. P. (1976). Empirical tests of multipliers for the prime modulus random number generator $\mathrm{X}_{i+1}=\mathrm{AX}_{i}\left(\bmod 2^{31}-1\right)$. In D. C. Hoaglin \& R. E. Welsch (Eds.), Proceedings of the Ninth In- terface Symposium on Computer Science and Statistics (pp. 178-183). Boston: Prindle, Weber \& Schmıdt.

Learmonth, G. P., \& LeWIS, P. A. W (1974). Statistical tests of some widely used and recently proposed uniform random number generators. In W J Kennedy (Ed.), Proceedings of the Seventh Conference on the Computer Science and Statistics Interface Ames, LA Iowa State University Press.

LEHMAN, R. S. (1977). Computer simulation and modeling Hillsdale, NJ: Erlbaum.

Lewis, P A. W., Goodman, A. S. \& Miller, J M. (1969) A pseudorandom number generation for the System 360. IBM Systems Journal, 8, 136-146.

LewIS, P. A. W., \& Orav, E. J. (1989). Simulation methodology for statisticians, operations analysts, and engineers (Vol. 1). Pacific Grove, CA: Wadsworth.

NORTON, D. W (1952). An empirical investigation of some effects of non-normality and heterogeneity of the F-distribution. Unpublished doctoral dissertation, State University of Iowa.

Ramberg, J. S., Dudewicz, E. J., Tadikamalla, P. R., \& Mykytka, E. F. (1979). A probability distribution and its uses in fitting data. Technometrics, 21, 201-214

Ramberg, J. S , \& Schmeiser, B. W (1972). An approximate method for generating symmetric random variables. Communications of the $A C M, 15,987-990$

RAMBERG, J. S., \& SCHMEISER, B. W. (1974). An approximate method for generating asymmetric random variables. Communications of the $A C M, 17,78-82$

SCHEFFE, H. (1959). The analysis of variance. New York. Wiley.

\section{NOTES}

1. The microcomputer laboratory was funded by an NSF-ILI grant, USE-8852194, awarded to Bates College by the National Science Foundation. The principal investigators were Georgia Nigro and Drake $\mathbf{R}$. Bradley of the Department of Psychology.

2. In this paper, the most recent release of Version 1.1 of DATASIM is described. It is specifically enhanced for conducting Monte Carlo research. The software is available at a nominal cost from Desktop Press, 90 Bardwell St., Lewiston, Maine, 04240 (telephone: $207-786-4113$ or 6180 ). DATASIM is designed to run on IBM-compatible PCs with $640 \mathrm{~K}$ of memory. A comparable version for the Macintosh is currently in development.

3. Here we focus exclusively on using DATASIM to conduct statistical simulations. DATASIM has three additional important applications, however: (1) It may be used as an automated homework-generating and answer-correcting system for students taking courses in statistics and research methods; (2) it may be used to conduct CAI demonstrations in class, as well as laboratory assignments out of class, which illustrate principles of sampling theory such as the central limit theorem; and (3) it may be used as a relatively comprehensive univariate statistical package that has excellent analysis of variance and analytical comparison subroutines. These applications, which are reviewed in greater detail by Bradley (1989a, 1989b), are documented fully in the DATASIM user manual (Bradley, 1988).

4. A variety of algorithms are available for generating data following non-normal distributions, such as the exponential. However, rather than build in different algorithms for every conceivable nonuniform distribution, DATASIM employs the generalized lambda distribution (GLD) developed by Ramberg and Schmeiser (1972, 1974) and Ramberg, Dudewicz, Tadikamalla, and Mykytka (1979). This four-parameter distribution may be used to approximate a wide variety of unimodal symmetric and nonsymmetric distributions. For an extended discussion of DATASIM's implementation of the GLD, see Bradley, (1989b, pp. 162-171) or Bradley (1988, pp. 63-68, 97-98). A GLD is defined in DATASIM by the LAMBDA command, and, once defined, it controls the generation of any simulated data. The theoretical distribution defined by LAMBDA may be plotted by entering the PDF command.

5. The use of a master seed means that the entire set of 3,000 data values needed for the simulation (three observations per sample for 1,000 samples) will be generated in sequence from this seed. Of course, prior to generating any particular dataset, the seed has an initial value based 
on the last update of LLRANDOMII. During repetitive simulation, the initial value of the seed for each dataset is displayed along with the results of any analyses requested (see the Appendix). Knowing the initial value of the seed allows one to regenerate the $n=3$ observations for a particular simulated dataset; knowing the master seed allows one to regenerate the data for all 1,000 simulated datasets. (Note that the initial value of the seed for the first simulated dataset is the same as that for the master seed.) DATASIM also provides an option for randomly sampling the seeds used to generate the datasets, instead of using seeds derived ultimately from one master seed.

6. We thank Melinda Harder, statistical consultant at Bates College, and Margaret Judge, senior honors major in mathematics and psychology, for recalculating the exact Type I error rates published by Box (1954) and Hsu (1938), respectively.

7. For example, for $\alpha=.05$, Box (1954, p. 299) reports an exact Type I error rate of .0403 when the $F$ test is applied to samples of $n_{1}=$ $3, n_{2}=5$, and $n_{3}=7$ observations drawn from populations with $\sigma^{2}$ in ratios of 1:2:3 (e.g., 10, 20,30). However, DATASIM consistently generates estimates of about .0320 for this same case. The recalculated exact value nums out to be .03257 . Similarly, Hsu $(1938$, p. 12) reports an exact Type $I$ error rate of .025 when a $t$ test is applied to samples of $n_{1}=5$ and $n_{2}=15$ drawn from populations with $\sigma^{2}$ in ratios of $1: 2$ (e.g., 10 vs, 20), whereas DATASIM generates estimates of around .0228 . The recalculated exact value is .02257 . Altogether, 4 of the 11 exact error rates reported by Box (1954) and 2 of the exact error rates reported by Hsu (1938) contain sufficiently large computational errors to produce significant differences when compared to DATASIM estimates. These differences disappear when the estimates are compared to the recalculated values.

8 . We have encountered only one systematic discrepancy with previous empirical work. Norton (1954) found that sampling from leptokurtic distributions produced inflated Type I error rates: In the two cases he investigated, the empirical error rates were .0783 and .0656 for a nominal level of $\alpha=.05$. When DATASIM is used to replicate these same conditions, we obtained deflated error rates of .0484 and .0403 , respectively. The latter finding is in fact consistent with research summarized by Glass et al. (1972, p. 273). We are presently attempting to locate the source of the discrepancy with Norton's work. The most likely explanation is that Norton made an error in specifying his leptokurtic distributions (Norton, 1989, personal communication), but, given the long lapse in time since the study, it is very difficult to pinpoint this precisely

\section{APPENDIX}

Repetitive Simulation with DATASIM: The One-Sample $z$ Test

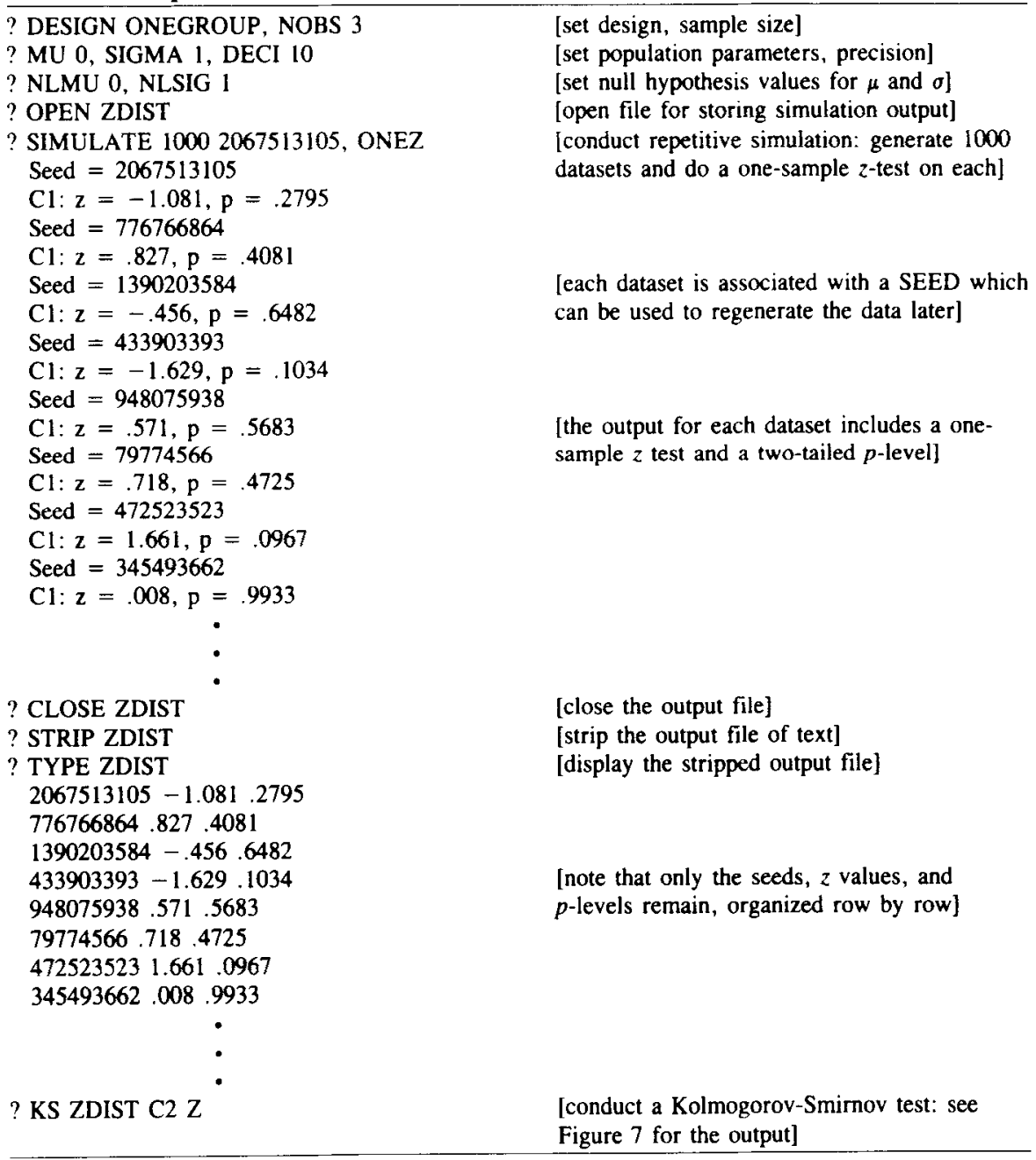

